\title{
Genetic insight and mapping of the pod constriction trait in Virginia-type peanut
}

\author{
Abhinandan S. Patil', Sigal Popovsky¹, Yael Levy'1, Ye Chu², Josh Clevenger², Peggy Ozias-Akins² and \\ Ran $\operatorname{Hovav}^{1 *}$ (D)
}

\begin{abstract}
Background: Pod constriction is an important descriptive and agronomic trait of peanut. For the in-shell Virginia marketing-type, this trait has commercial importance as well, since deeply constricted pods have a tendency to break, which makes them unmarketable. Classical genetic studies have indicated that pod constriction in peanut is controlled by one to four genes, depending on the genetic background. In all of those studies, pod constriction was evaluated visually as opposed to quantitatively. Here, we examined the genetic nature of this trait in the Virginia-type background. Our study involved 195 recombinant inbred lines ( $\left.F_{7} R I L s\right)$ derived from two closely related cultivars that differ in their degree of pod constriction. Pod constriction was evaluated visually and quantitatively in terms of the pod constriction index $(\mathrm{PCl})$, calculated as the average ratio between the pod's waist and shoulders.
\end{abstract}

Results: ANOVA and genetic parameters for $\mathrm{PCl}$ among the $\mathrm{F}_{7} \mathrm{RILs}$ in three blocks showed very significant genotypic effect $(p(F)<0.0001)$ and high heritability and genetic gain estimates $(0.84$ and 0.52 , respectively). The mean $\mathrm{PCl}$ values of the different RILs had a bimodal distribution with an approximate 1:1 ratio between the two curves. Pod constriction was also determined visually (VPC) by grading the degree of each RIL as 'deep' or 'slight'. The $x^{2}$ test was found to not be significantly different from a 1:1 ratio $(p=0.79)$ as well. SNP-array-based technology was used to map this trait in the RIL population. A major locus for the pod constriction trait was found on chromosome B7, between B07_120,287,958 and B07_120,699,791, and the best-linked SNP explained 32\% of the total variation within that region. Some discrepancy was found between the SNPs original location and the genetic mapping of the trait.

Conclusion: The trait distribution and mapping, together with data from $F_{1}$ and $F_{2}$ generations indicate that in this background the pod constriction is controlled by a major recessive gene. The identity of loci controlling the pod constriction trait will allow breeders to apply marker-assisted breeding approaches to shift allelic frequencies towards a slighter pod constriction and will facilitate future effort for map-based gene cloning.

Keywords: Virginia-type peanut, Pod constriction, Recombinant inbred lines, SNP-Array, Genetic mapping, Physical mapping

\section{Background}

Peanut (Arachis hypogaea L.) is known for the incongruity between its very narrow genetic polymorphism and the great phenotypic diversity among peanut cultivars $[1$, 2]. Domesticated peanut is allopolyploid $(2 n=4 \times=40)$ that is composed from two diploid species Arachis

\footnotetext{
* Correspondence: ranh@agri.gov.il

${ }^{1}$ Department of Field Crops, Institute of Plant Sciences, Agriculture research organization -the Volcani Center, HaMakkabbim Road, P. O. Box 15159, 7505101 Rishon LeZiyyon, Israel

Full list of author information is available at the end of the article
}

duranensis and Arachis ipaensis, named A and B genome. As in many polyploid species, cultivated peanut has experienced a genetic bottleneck which, superimposed with the effects of domestication and self-pollinating system, has greatly narrowed the genetic diversity and limited DNA polymorphism among subsequently derived Arachis forms [2]. The phenotypic diversity can be seen in peanut pods, which vary widely in their size, structure and texture. One of these highly variable traits, pod constriction, is an important descriptive trait used to distinguish between peanut 
market-types [3]. For example, Valencia (fastigiata) types are recognized by their very slight pod constriction; whereas Virginia (hypogaea) and Spanish (vulgaris) types usually have slight-to-moderate pod constriction. The hirsuta subspecies ( $A$. hypogaea ss. hirsuta) characterized by deep to very deep pod constriction, particularly between the second and third seed segments. Pod constriction can also vary considerably within peanut types, especially within the Virginia-type group, in which the degree of pod constriction ranges from very deep to very slight.

Pod constriction is also an important biological and commercial trait. It influences seed development, since pods with very slight or no constriction usually bear tightly packed, flattened seeds that are in direct contact with their neighbors [4]. This may cause damage to the embryos and also increase the amount of seed-splitting during the shelling process. On the other hand, highly constricted pods are undesirable as well, especially for the in-shell industry, because they tend to carry dirt that detracts from the pod's final tint. More importantly, they tend to split during the harvesting and sorting process, rendering the final product unmarketable.

Due to its commercial importance, the genetic nature of pod constriction has been the subject of several studies. In fact, pod constriction is one of the oldest genetically inspected traits, as it was one of the seven traits that Mendel [5] studied in pea. Mendel hypothesized that a single gene controls the pod constriction trait, in which the "inflated" pod phenotype is dominant over the "constricted" pod phenotype. Pod constriction is the only one of Mendel's genetic traits that has not yet been cloned and this trait has received less research attention than any of the other traits he studied $[6,7]$. In peanut, several classical studies were performed to address the inheritance of pod constriction and those studies led to the proposals of a two-gene model [8] and a three-gene model [9] in which slight constriction is dominant over deep constriction. A four-gene model (three unlinked nuclear loci and one cytoplasmic locus) was also proposed [10]. In that model, the deep constriction is dominant over slight constriction. A more recent study involving Spanish-type peanuts [11] reported that a single gene controls pod constriction, with deep constriction dominant over slight constriction. Also, the inheritance of pod constriction was analyzed by using a cross between a Spanish-type variety and its narrow-leaf mutant [12, 13], suggesting that pod constriction is under trigenic control (i.e., any two of the three complementary dominant genes lead to constricted pods). The identification of regions controlling the pod constriction trait will lead to fine mapping efforts to further the understanding of the genes controlling the trait.

In the present study, a recombinant inbred lines (RILs) population was analyzed to investigate the genetic nature of the pod constriction trait of peanut. The population is based on a cross between very closely related Virginia marketing-type cultivars that differ in their pod constriction. As mentioned above, pod constriction is an important trait in the Virginia-type in-shell industry. Yet, there is little information in the literature regarding pod constriction among Virginia-type germplasm. We evaluated pod constriction visually (VPC) and also quantitatively, by measuring the pod constriction index (PCI) in a random sample of pods. In addition, SNP-array-based technology $[14,15]$ was used to map the pod constriction, providing new insight into the genetic nature of this trait in peanut.

\section{Results}

Variance and genetic analyses of pod constriction among the RIL population

Variance and genetic parameters for PCI among 195 $\mathrm{F}_{7}$ RILs in three blocks are presented in Table 1. As shown, the genotypic effect of the RILs on PCI was very significant $(p(F)<0.0001)$, while the effect of the block was non-significant $(\mathrm{p}(\mathrm{F})=0.87)$. In the agreement, the heritability and genetic gain estimates were

Table 1 ANOVA and genetic parameters for PCl among $195 \mathrm{~F}_{7}$ RILs in three blocks

\begin{tabular}{|c|c|c|c|c|c|}
\hline Source of variation & Degrees of freedom & Sum of Squares & Mean Squares & F ratio & Probability $(F)$ \\
\hline Block & 2.00 & 0.00 & 0.00 & 0.19 & 0.87 \\
\hline RIL & 194.00 & 24.29 & 0.13 & 19.31 & $<0.0001$ \\
\hline Error & 388.00 & 2.52 & 0.006 & & \\
\hline \multicolumn{6}{|l|}{ Genetic parameters } \\
\hline \multicolumn{2}{|l|}{ ECV (\%) } & \multicolumn{4}{|l|}{11.04} \\
\hline \multicolumn{2}{|l|}{ GCV (\%) } & \multicolumn{4}{|l|}{27.26} \\
\hline \multicolumn{2}{|l|}{ PCV (\%) } & \multicolumn{4}{|l|}{29.41} \\
\hline \multicolumn{2}{|c|}{$h^{2}$ (Broad Sense) (\%) } & \multicolumn{4}{|l|}{83.92} \\
\hline \multicolumn{2}{|c|}{ Genetic Advancement (5\%) } & \multicolumn{4}{|l|}{52.05} \\
\hline
\end{tabular}

ECV; GCV; PCV- environmental, genetic and phenotypic coefficient of variation, respectively. $\mathrm{h}^{2}$ - heritability 
high (0.84 and 0.52, respectively). Accordingly, the further genetic and mapping analyses were done on the average PCI values.

The distribution of the average PCI scores of the 195 RILs is presented in Fig. 1. Average PCI varied between 0.39 and 1.08 . Yet, as shown, PCI was not distributed normally, but instead appeared to have a clearly bimodal distribution with some small overlap between the two curves. The median PCI was 0.86 with a small tendency toward the higher PCI values, suggesting an approximate 1:1 ratio between the two curves. The parental line values were located within each of the curves. Some deviation of the lower values from the value observed for cv. Hanoch was noted, illustrating an over-representation of the extra-deep pod constriction phenotype in the RIL population.

Pod constriction was also determined visually (VPC) by grading the degree of pod constriction of each RIL as deep (like 'Hanoch') or slight (like 'Harari'). Out of the 195 RILs, $100 \mathrm{~F}_{7}$ RILs were rated as "deep" and 95 RILs were rated as "slight" (Additional file 1: Figure S1; Table 2). On the basis of the expected and observed frequencies of $\mathrm{VPC}$, the $\chi^{2}$ test was found to not be significantly different from a 1:1 ratio $(p=0.79)$. VPC was also evaluated among Hanoch $\times$ Harari based $F_{1}$ and $F_{2}$ populations (a detailed description of those populations can be found in Kayam et al. [16]). VPC segregated at a rate of 5:0 for slight: deep among the $\mathrm{F}_{1}$ plants and at a rate of approximately $3: 1(p$ $=0.39$ ) among the $F_{2}$ generation (Table 2).

The association between VPC and PCI among the RILs was inspected (Fig. 2). In general, a strong relationship was observed between VPC and PCI, as reflected by the significant difference $\left(\mathrm{p}(\mathrm{t})<0.0001 ; \mathrm{R}^{2}=0.84\right)$ between the average PCIs of the deep (0.56) and slight
(0.93) VPC groups. Yet some points were outlined on the graph, especially from the deep VPC group, presenting high PCI values.

\section{Mapping of VPC and PCI}

Physical localization of VPC and PCI in the peanut diploid genomes was done by TASSEL. Prior to trait-mapping, the adjusted association of 2882 SNPs with the pod constriction traits (VPC and PCI) was examined using quantile-quantile plots (Additional file 1: Figure S2). The quantile-quantile plots of both VPC and PCI were found to deviate from a uniform distribution, indicating that significant associations exist between SNPs and both traits. These associations were further confirmed by the physical mapping of VPC and $\mathrm{PCI}$ (Fig. 3). As shown in the plots, most of the SNPs associated with VPC and PCI were found in the same region at the end of chromosome B7. With the threshold of $-\log _{10}(\mathrm{p}) \geq 4.76$ (red line in Fig. 3a, b), 21 and 15 SNPs were significantly linked with VPC and PCI, respectively. One significant exception was a VPC-associated SNP found on chromosome 8 (Fig. 3a). The $-\log _{10}(\mathrm{p})$ of the significant SNPs for VPC ranged from 15.20 to 4.85 and the total phenotypic variation explained by SNP markers $\left(R^{2}\right)$ ranged from 0.32 to 0.11 (Additional file 1: Table S1). PCI $-\log _{10}(\mathrm{p})$ values ranged from 13.79 to 4.78 and total phenotypic variation explained by SNP markers $\left(R^{2}\right)$ ranged from 0.30 to 0.11 .

Genetic mapping of VPC and PCI was performed to evaluate the original locations of the SNPs by their recombination rate in the population and particularly to validate the order of the SNPs within chromosome B7. A total of 19 linkage groups were generated with a $1570.16 \mathrm{cM}$ map (Additional file 2: Table S2). Notably, the linkage groups of the genetic map did not match the

\begin{tabular}{|c|c|c|}
\hline \multicolumn{2}{|c|}{ Quantiles } & $\mathrm{PCl}$ \\
\hline $\begin{array}{l}100.0 \% \\
99.5 \%\end{array}$ & maximum & $\begin{array}{l}1.08 \\
1.08\end{array}$ \\
\hline $97.5 \%$ & & 0.9905 \\
\hline $90.0 \%$ & & 0.96 \\
\hline $75.0 \%$ & quartile & 0.92 \\
\hline $50.0 \%$ & median & 0.86 \\
\hline $25.0 \%$ & quartile & 0.53 \\
\hline $10.0 \%$ & & 0.49 \\
\hline $2.5 \%$ & & 0.45 \\
\hline $0.5 \%$ & & 0.39 \\
\hline $0.0 \%$ & minimum & 0.39 \\
\hline
\end{tabular}

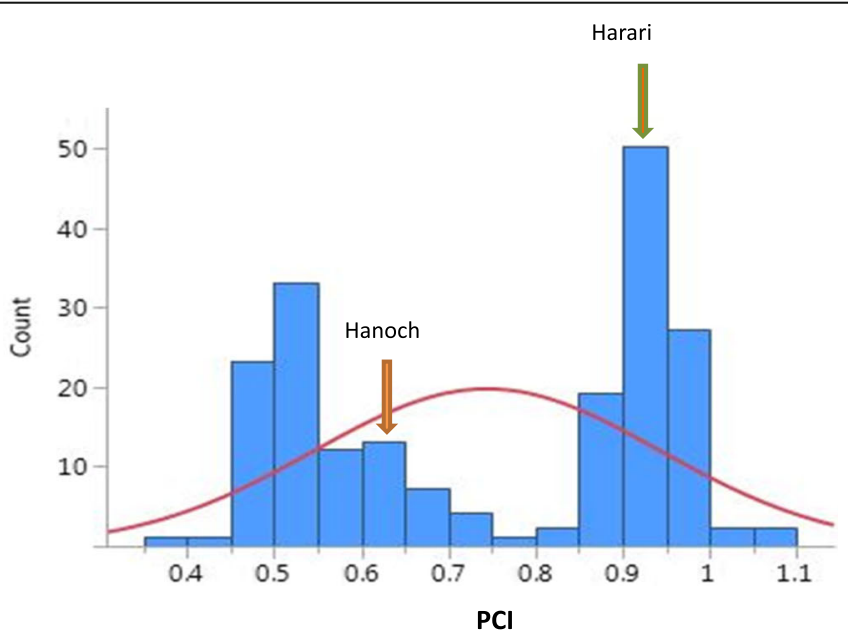

Fig. 1 Distribution of the mean pod constriction index (PCI) trait in 195 RILs derived from the Hanoch X Harari cross. Count = number of RILs in this category 
Table 2 Inheritance of pod constriction as determined visually (VPC) among the $F_{1}, F_{2}$ and $F_{7}$ progeny of 'Hanoch' $\times$ 'Harari'

\begin{tabular}{llllllll}
\hline Generation & \multicolumn{2}{l}{ Phenotype } & & Expected ratio & Observed ratio & X2 value & $p$ value \\
\cline { 2 - 4 } & Total & Deep constriction & Slight constriction & & & \\
\hline$F_{1}$ & 5 & - & 5 & 195 & $3: 1$ & - & - \\
$F_{2}$ & 273 & 78 & 95 & $1: 1$ & $1: 2.5^{*}$ & 0.74 \\
$F_{7}$ & 195 & 100 & $954: 1^{*}$ & 0.07 & 0.79 \\
\hline
\end{tabular}

$d f=1$. ${ }^{*}$ The observed ratio is not significantly different from the expected ratio at $p=0.05$

$F_{1}$ and $F_{2}$ plants were grown as described at [16]

original SNPs locations; many SNP markers originally considered to be A- or B-derived [14, 15] were mapped to the same linkage group (Additional file 2: Table S2). VPC and PCI were mapped to a single QTL at linkage group 15 at closely linked SNPs between markers B07_120,287,958 and B07_120,699,791, with best LOD scores of 10.17 and 9.95, respectively (Fig. 4). In contrast to most of the linkage groups, the order of the SNPs within this linkage group was almost completely in accordance with their expected physical order on chromosome B7, with only minor discrepancies and the inclusion of a number of SNPs from chromosomes A7 and A8. The SNP marker AX176792556, which was originally located at A8_1053912, and was found to be significantly linked to VPC (Fig. 3a), was found within linkage group 15.

\section{Discussion}

In the current study, the inheritance pattern of the pod constriction trait was investigated in Virginia-type peanut. Pod constriction is a very important trait in the in-shell market. Occasionally breeders choose not to commercialize highly performing breeding lines for that market only because of excessive pod constriction, which causes pods to break during harvest, handling and shipping. The identity of genes controlling the pod

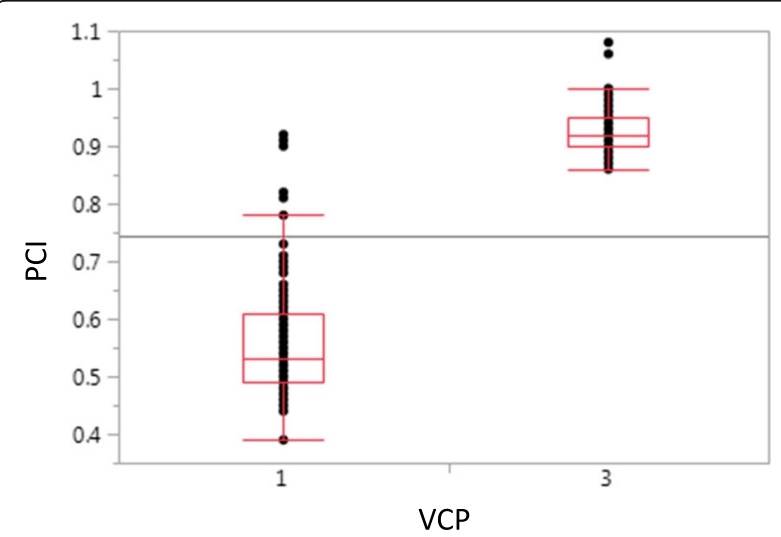

Fig. 2 Comparison of quantitative and visual pod constriction of $195 \mathrm{~F}_{7}$ RILs. Quantitative pod constriction is the constriction index (PCl) based on quantitative measurements of the waist and shoulders of the peanut pod. Visual pod constriction (VPC) was rated on a scale of 1 (deep) to 3 (slight) constriction trait will allow breeders to apply marker-assisted breeding approaches to develop new genotypes with slighter pod constriction, alleviating the commercial industry of potential issues with splitting and aesthetics presented by some cultivars like cv. Hanoch. We used closely related Virginia-type peanut germplasm to study this trait against a homogenous genetic background. Therefore, in contrast to earlier studies based on crosses of relatively distantly related parents with extreme phenotypes (i.e., very slight vs. very deep pod constriction), the current study focused on less extreme phenotypes (i.e., slight vs. deep pod constriction).

Indeed, the result of 1:1 segregation of the trait indicates that against this genetic background, pod constriction may be controlled by a major gene, regardless of whether it was phenotyped visually or quantitatively. This is in contrast to the findings of most other pod constriction inheritance studies, which have indicated that there are several genes that control this trait in peanut $[8-10,12]$. The explanation for this contrast may be the genetic material of the current study that is differing only in one locus while the other possible loci were not detected as an effect of selection (since two closely related cultivars were crossed). The slight pod constriction phenotype was dominant over the deep pod constriction phenotype, as shown by the segregation of this trait in the early $F_{1} / F_{2}$ generations. Interestingly, while the difference between the PCI values of the two parental lines was not particularly great, many RILs had significantly lower PCI values that indicate very deep pod constriction. This unexpected deep pod constriction phenotype suggests a possible environmental effect on the trait, the epistatic contribution of additional minor genes or a combination of those two factors.

A common characteristic of all of the above-mentioned studies is that they were based on visual, as opposed to quantitative evaluations of pod constriction. Here, pod constriction was evaluated both visually, by inspecting and grading the entire sample, and quantitatively, by measuring the ratio between the waist and shoulders (PCI) of randomly selected pods. It was found that, in general, no large differences exist between the two methods $\left(R^{2}=\right.$ 0.84 ) and data sets collected using both methods suggested that pod constriction is controlled by a major gene and, in both analyses, that putative gene was mapped to 


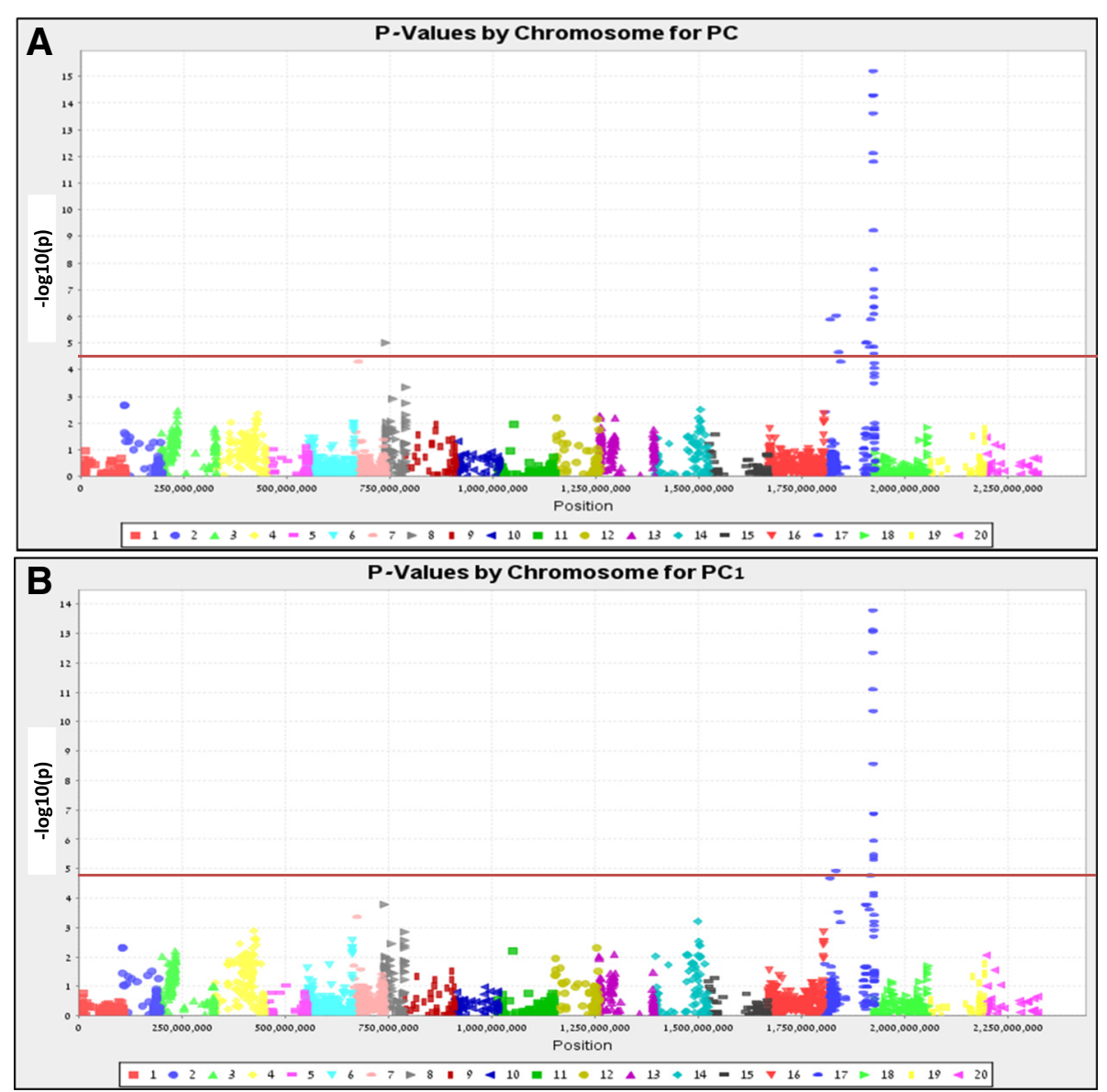

Fig. 3 Microarray-based Manhattan plot showing a significant association between the SNPs for VPC (a) and PCI (b). The relative densities of SNPs physically mapped on 20 chromosomes of the peanut genome are plotted along the $x$-axis. $1-10=$ genome A (1-10); 11-20=genome B (1-10). The $-\log _{10}(p)$ for the association between SNP loci and pod constriction are plotted along the $y$-axis. The red line indicates the cut-off for significance at $p \leq 10^{-4.76}$

the same genomic location. Since these ratings were similar, the visual rating for pod constriction would be more efficient while evaluating many breeding plots. Yet, our results (as shown in Fig. 2) indicate that quantitative measurements of PCI may be more accurate than visual assessments. Therefore, the quantitative measurement should be the preferred method for tracking pod constriction in different backgrounds as well as for the identification of additional loci controlling the pod constriction trait in peanut.

SNP-array-based technology was used to map the VPC trait in the RIL population and identified a single locus for the VPC trait on chromosome B7. A large discrepancy was found between the genetic map and the original SNP localization on the A and B sub-genomes, since many A- and B-originating SNPs were assigned to the same linkage group. In some cases it was difficult to pair the linkage group to its corresponding physical chromosome. This observation, together with those of other studies that have used this SNP array for trait-mapping, indicates that it is important to re-address the positions of the SNPs by genetic mapping. Fortunately, linkage group 15 was constructed mostly from SNPs from B7, enabling us to locate PC and PCI in a small linkage group between markers B07_120,287,958 and B07_120,699,791. Some discrepancy was also found between the SNP original position and the genetic map of the traits within linkage group 15, indicating that there may be some microsyntenic differences in this region between the diploid and the tetraploid species.

An interesting outcome of the mapping analysis was that VPC and PCI were mapped to a single locus, but with relatively low $-\log _{10}(\mathrm{p})$ and a relatively low LOD score. The best-linked SNP explained 32\% of the total variation and a LOD score of $\sim 10$ (for VPC), which could be considered low for a major gene effect. This may be due to insufficient resolution of the SNP markers in this region. For example, although the genetic map showed that the pod constriction locus is located between B07_120,287,958 and B07_120,699,791, a large gap in SNP density was noted just 


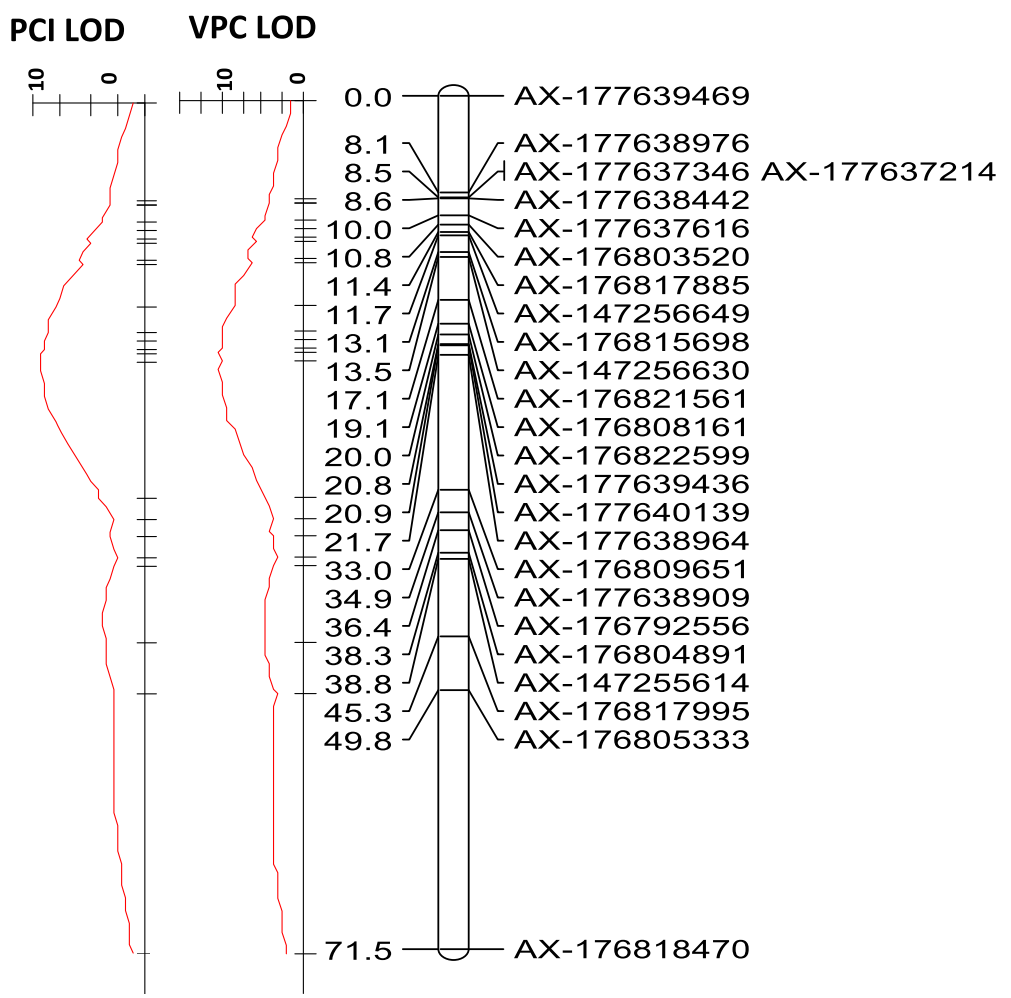

Fig. 4 Genetic mapping of PCl and VPC on linkage group 15 and comparison to the physical position. Distances between markers are indicated in $\mathrm{CM}$, to the left of the linkage group. LOD scores for the links between the SNPs and the traits are presented in the red graph on the left

below B07_120,287,958, with no downstream markers until B07_114704092 (Fig. 4). It is also possible that more minor loci also affect pod constriction in this background, which was not diagnosed significant in our relative strict Bonferroni cutoff of $p$-value $\leq 10^{-4.76}$ (Fig. 3).

According to the assumption that the VPC locus is indeed located within this $\sim 400-\mathrm{kb}$ region, an additional analysis of candidate genes was performed. Fifteen gene models were collected from this segment (Additional file 1: Table S3) using the peanut genome database [17]. Most of the genes did not match any of the gene products that have been associated with pod shape or fruit shape in the literature. However, one gene, a MADS-box transcription factor 1 located in the middle of this linkage group, may play a role in controlling VPC, since we speculate that the new pod shape should be controlled by multiple gene expression factors. Interestingly, this gene was previously found to be expressed solely in the pistil [18]. We hypothesize that the controller of pod constriction would be expressed much earlier in pod development, possibly even during the development of the female gametophyte or the fertilization process. Moreover, BLASTing this gene to the NR_PubMed database yielded two best-hits that are both Agamous-like MADS-box proteins, AGL104 and AGL11, with 70\% and $67 \%$ identity, respectively. AGL11 is a transcription factor that is required for the maternal control of the formation of endothelium, which is the tissue that surrounds the seeds during their development [19]. This gene should be studied further, to examine its hypothetical role as a controller of pod constriction.

\section{Conclusion}

The trait distribution and mapping that are presented in the current study, together with data from $F_{1}$ and $F_{2}$ generations, indicate that in this background the pod constriction is controlled by a major recessive gene. The identity of loci controlling the pod constriction trait will allow breeders to apply marker-assisted breeding approaches to shift allelic frequencies towards a slighter pod constriction and will facilitate future effort for map-based gene cloning.

\section{Methods}

Plant material and mapping populations

A recombinant inbred line population $\left(\mathrm{F}_{7} \mathrm{RIL}\right)$ that was derived from the cross of cv. Hanoch and cv. Harari, two closely related Israeli Virginia-type cultivars, was developed [20] and used for the study. 'Hanoch' has been the leading Israeli in-shell peanut cultivar for over two decades. It has long, smooth pods and a unique flavor, which made it very popular in the market. A major drawback of this cultivar is 
its relatively deeply constricted pod, which contributes to its "long shape" appearance, but also makes it vulnerable to pod-splitting. 'Harari', another Virginia-type cultivar, is characterized by smaller pods with moderate-to-slight pod constriction.

RILs were planted in April 2016 on broad bed furrow (two rows, $75 \times 40 \mathrm{~cm}$ ) in Nirim Magar, a location in southern Israel that is characterized by fine-sandy loam soil. Each RIL was replicated three times (randomized complete block design), with 16 plants for each RIL in each block. Plots were maintained under full-irrigation conditions and all recommended agronomic practices were carried out as described [20]. 'Hanoch' and 'Harari' plants were also grown as controls. Pods from each RIL and the parental cultivars were harvested manually by randomly collecting 300 sound and fully mature pods from the plants of each plot. In addition to the RIL population, $F_{2}$ generation that was derived from the same cross was validated for PC in field trial based on individual plants. All field procedures for this population were previously described [16].

\section{Phenotyping of the pod constriction trait}

For each RIL, the level of constriction was determined in two ways. First, the phenotype was determined visually by inspecting the entire sample and grading the pod constriction of each of the $F_{7}$ RILs as "deep" or "slight" (groups 1 or 3 in Additional file 1: Figure S1). Second, a random 30 pods were sampled from each RIL and a Vernier caliper was used to measure the breadth of the top and bottom shoulders of each pod, as well as the width of each pod's constricted (waist) area. The pod constriction index (PCI) was calculated from mean values using the following formula:

$$
P C I=\frac{\text { Waist width }(\mathrm{mm})}{(\text { Top }+ \text { bottom shoulder width }(\mathrm{mm})) / 2}
$$

The measured and visual assessments of the pod constriction of the 195 members of the $F_{7}$ RIL population, as well as the frequency distribution of the pod constriction ratios, were analyzed using $\mathrm{JMP}^{\circ}$ version 10 (SAS Institute; Cary, NC, USA). A chi-square $\left(\chi^{2}\right)$ test was used to study the segregation pattern of pod constriction traits.

\section{Genotyping and SNP filtering}

Young leaflets from each $\mathrm{F}_{7}$ RIL were collected and genomic DNA was extracted using DNeasy Plant Mini Kit (Qiagen; Hilden, Germany). Precise DNA quantification was carried out with Qubit (Invitrogen; CA, USA) and the samples were diluted to $30 \mathrm{ng} / \mu \mathrm{L}$ according to the Affymetrix guidelines. The second edition of the Axiom_Arachis array (Axiom_Arachis2) designed by Ozias-Akins lab, which is an improved version of the previously reported $58 \mathrm{k}$
Axiom_Arachis SNP-array [14, 15], was used with 47,837 SNPs to genotype the 197 individuals (two parents and $195 \mathrm{~F}_{7}$ RILs). Genotyping data were analyzed by the Axiom analysis suite. The software output of the genotyping data in five categories based on the quality of signal clustering i.e. polyhighresolution (three clusters with good separation, major allele, minor allele and heterozygous state, minor allele at least detected two times), nominorhom (two clusters, minor allele is not present in homozygous state), monohighresolution (marker monomorphic, minor allele with zero or one copy), CallRateBelow Threshold (SNP call rate is below $90 \%$, but other cluster properties are above) and Off-Target Variant categories (unexpected new alleles). Genotyping data was only extracted from polyhighresolution and nominorhomo categories since monohighresoultion class did not contain any polymorphic markers and most of the markers in the CallRateBelow Threshold and Off-Target Variant categories were ambiguous with signal clustering. The polymorphic homozygous SNPs (AA and $\mathrm{BB})$ and polymorphic heterozygous SNPs (AA or BB and $\mathrm{AB})$ were retained with $65-35 \%$ call-rate frequencies among the RILs. Two thousand eight hundred and eighty-two polymorphic SNPs were retained for further analyses.

\section{Physical localization of pod constriction trait in the peanut diploid genomes}

The pod constriction trait was mapped based on the Axio$\mathrm{m} \_$Arachis2 SNP markers. TASSEL 5 [21] was used to test the association of the pod constriction trait with each of the 2882 SNPs across the peanut diploid genomes [22]. First, a general linear model was run and all of the probabilities generated in the association runs were transformed by $-\log _{10}(\mathrm{p})$. Scores for each chromosome were then inspected in Manhattan plots to determine whether the SNPs reached the significance threshold for visual and quantitative measurements of pod constriction. The critical $p$-values for assessing the significance thresholds for the SNPs were corrected for multiple comparisons based on the Bonferroni method [23]; which gave the adjusted p-value of - $\log 10$ $(\alpha / n) ; \alpha=$ significance level and $n=$ the number of markers (2882 SNPs). The Bonferroni adjusted cut-off for accepting thresholds was set to $-\log _{10}(\mathrm{p}) \geq 4.76$, which corresponds to an experiment-wise error rate of 0.05 .

\section{Genetic map construction and QTL analysis}

Genetic maps were constructed using Join-Map 4.1 software [24]. From the error-free datasheet, a population node was created for 2849 loci (33 duplicated SNPs were excluded from the total 2882 SNPs) and 195 "individuals" (RILs). The multipoint maximum-likelihood mapping algorithm [25] was used to calculate locus genotype frequency, remaining only SNPs with chi-square p-value $\leq 0.05$ ( 1 degree of freedom). Resulting the chi-square test, 723 loci 
were excluded for exceeding the threshold. Out of 4,056,976 pairs, 6112 were excluded due to loci-similarity. Groupings were established according to an independent LOD that started at 2.0 and ended at 10. Map distances were estimated using Haldane's (1919) mapping function. QTL mapping analyses of the $195 \mathrm{~F}_{7}$ RILs were performed using MapQTL 6 [26] for the visual (VPC) and quantitative (PCI) pod constriction traits. Loci were detected by interval mapping using significance threshold levels derived from permutation tests. A LOD score of 2.5 with 1000 permutations was used to confirm the presence of a putative QTL.

\section{Statistical analysis}

The analysis of variance was carried out according to the standard procedure described by Panse and Sukhatme [27]. The statistical model for the randomized block design used for the analysis of variance was:

$$
Y_{i j}=\mu+r_{i}+\mathrm{g}_{j}+\delta_{i j}
$$

In that model, $Y_{i j}$ is the response of the $j^{\text {th }}$ RIL in the $i^{\text {th }}$ block, $\mu$ is the general mean, $r_{i}$ is the effect of the $i^{\text {th }}$ block, $g_{j}$ is the effect of the $j^{\text {th }}$ RIL and $\delta_{i j}$ is the random residual error associated with the $j^{\text {th }}$ genotype in the $i^{\text {th }}$ replication.

The genetic variability, heritability and genetic advance parameters were calculated in a similar method as previously described [20]. Briefly, environmental, genotypic and phenotypic coefficients of variation (ECV, GCV and PCV) for existing in traits were estimated using the formula:

$$
\begin{aligned}
& E C V(\%)=\frac{\sqrt{\hat{\sigma}_{\mathrm{e}}^{2}}}{\overline{\mathrm{X}}} \times 100, \quad G C V(\%)=\frac{\sqrt{\hat{\sigma}_{g}^{2}}}{\overline{\mathrm{X}}} \times 100, \\
& \operatorname{PCV}(\%)=\frac{\sqrt{\hat{\sigma}_{p}^{2}}}{\overline{\mathrm{X}}} \times 100
\end{aligned}
$$

wherein, $\sigma_{p}^{2}, \sigma_{g}^{2}$, and $\sigma_{e}^{2}$ are the phenotypic, genotypic and environmental variance components [20], respectively, and $\mathrm{X}$ is the general mean.

Heritability $\left(h^{2}\right)$ in broad sense was calculated according to the formula:

$$
h^{2}=\frac{\hat{\sigma}_{g}^{2}}{\hat{\sigma}_{p}^{2}} \times 100
$$

The expected genetic advance (GA) was measured using the formula:

$$
G A=\frac{\mathrm{V}_{\mathrm{g}}}{\mathrm{V}_{\mathrm{p}}} \times \sqrt{\mathrm{V}_{\mathrm{p}}} \times \mathrm{k},=\frac{\mathrm{V}_{\mathrm{g}}}{\sqrt{\mathrm{V}_{\mathrm{p}}}} \times \mathrm{k}
$$

In that formula, $V_{g}$ is the genotypic variance, $V_{p}$ is the phenotypic variance and $k$ is the selection differential (constant; i.e., 2.06 at $5 \%$ selection intensity).

\section{Additional files}

Additional file 1: Figure S1. Visual pod constriction descriptors for peanut. Cv. Hanoch and Harari belong to Deep (1) and Slight-Moderate (3) groups, respectively. Figure S2. Quantile-quantile plot of visually assessed pod constriction (VPC) and mean pod constriction index (PCl) values for $195 \mathrm{~F}_{7}$ RILs. Table S1. Significant SNP markers associated with visual (VPC) and quantitative (PCl) assessments of the pod constriction trait. Table S3. List of gene models within the B07:120287958. .

120,699,791 genomic segment. (PDF 398 kb)

Additional file 2: Table S2. Peanut linkage groups constructed based on $195 \mathrm{~F}_{7}$ RILs. (XLSX $75 \mathrm{~kb}$ )

\section{Abbreviations}

PCl: Pod constriction index; QTL: Quantitative trait locus; RIL: Recombinant inbred line; VPC: Visual pod constriction

\section{Acknowledgments}

The authors would like to thank Ilan Hedvat and Oren Buchshtab for their help in managing the field trial.

\section{Funding}

This study was funded by the Israeli Ministry of Agriculture (grant no. 20-100021) and an Israel-USA Bilateral Agriculture Research and Development (BARD) grant (grant no. IS-5020-17). The grant does not interfere with the scientific side of the study, so it does not have any role in the design of the study and collection, analysis, and interpretation of data and in writing the manuscript.

\section{Availability of data and materials}

All the supporting data was included in additional files.

\section{Authors' contributions}

ASP, was responsible for the project, and was a major contributor in writing the manuscript; SP assisted in construction of the genetic map; $Y L$ phenotyped the PC trait; YC, JPC and PO were responsible for the SNP-array managing and assisted in data analysis; $\mathrm{RH}$ cogitated the research and was a major contributor in writing the manuscript. All authors read and approved the final version of the manuscript.

\section{Ethics approval and consent to participate}

The experimental research complies with institutional (ARO) guidelines for plant material. The source of all plant materials is from Dr. Ran Hovav laboratory at ARO, Israel. The seeds for all lines are stored at Israeli Gene Bank (https://igb.agri.gov.il/web/index.php); (accession number -101000) and are available for distribution. No field permissions were necessary to collect the plant samples in the study.

\section{Consent for publication}

Not applicable.

\section{Competing interests}

The authors declare that they have no competing interests.

\section{Publisher's Note}

Springer Nature remains neutral with regard to jurisdictional claims in published maps and institutional affiliations.

\section{Author details}

'Department of Field Crops, Institute of Plant Sciences, Agriculture research organization -the Volcani Center, HaMakkabbim Road, P. O. Box 15159,

7505101 Rishon LeZiyyon, Israel. '2Department of Horticulture and Institute of Plant Breeding, Genetics and Genomics, The University of Georgia, Tifton, GA 31793, USA. 
Received: 21 May 2018 Accepted: 13 September 2018

Published online: 19 October 2018

\section{References}

1. Kochert G, Halward T, Branch WD, Simpson CE. RFLP variability in peanut (Arachis hypogaea L.) cultivars and wild species. Theor Appl Genet. 1991;81: 565-70.

2. Moretzsohn MC, Gouvea EG, Inglis PW, Leal-Bertioli SCM, Valls JFM, Bertioli DJ. A study of the relationships of cultivated peanut (Arachis hypogaea) and its most closely related wild species using intron sequences and microsatellite markers. Ann Bot London. 2013:111:113-26.

3. Pittman RN. United States peanut descriptors. Washington DC: U.S. Department of Agriculture, Agricultural Research Service; 1995.

4. Smartt J. The groundnut crop: a scientific basis for improvement. London: Springer Science \& Business Media; 2012.

5. Mendel G. Versuche über Pflanzen-Hybriden. Verhandlungen des Naturforschenden Vereines in Brünn, IV. Brünn: Verlage des Vereines; 1886. p. 67-112.

6. Reid JB, Ross JJ. Mendel's genes: toward a full molecular characterization. Genetics. 2011:189:3-10.

7. Smykal P. Pea (Pisum sativum L.) in biology prior and after Mendel's discovery. Czech J Genet Plant. 2012;50:52-64.

8. Hunter $\mathrm{H}$, Leake HM. Recent advances in agricultural plant breeding Philadelphia: Blakiston; 1933.

9. Hassan MA. Genetic, floral, biological and maturity studies in groundnut. Ranchi : Ranchi Agricultural College, Ranch University; 1964

10. Coffelt TA, Hammons RO. Inheritance of pod constriction in peanuts. J Hered. 1974:65:94-6.

11. Bera SK, Das PK. Inheritance of pod beak and pod constriction in groundnut (Arachis hypogaea L.). Curr Agric Res. 1998;11:1-3.

12. Pattanashetti SK, Gowda MVC, Girija X. Inheritance of morphological traits and pod features in groundnut (Arachis hypogaea L.). Ind J Genet PI Br. 2008;68:157-62

13. Hake AA, Shirasawa K, Yadawad A, Sukruth M, Patil M, Nayak SN, Lingaraju S, Patil PV, Nadaf HL, Gowda MVC, Bhat RS. Mapping of important taxonomic and productivity traits using genic and non-genic transposable element markers in peanut (Arachis hypogaea L.). PLoS One. 2017;12(10).

14. Clevenger J, Chu Y, Chavarro C, Agarwal G, Bertioli DJ, Leal-Bertioli SCM, et al. Genome-wide SNP genotyping resolves signatures of selection and tetrasomic recombination in peanut. Mol Plant. 2016a;10:309-22.

15. Pandey M, Agarwal G, Kale S, Clevenger J, Nayak S, Sriswathi1 M, et al. A powerful and high density genotyping tool for peanut genetics and breeding: development and evaluation of 58K Axiom_Arachis SNP array. Sci Rep. 2016:7.

16. Kayam G, Brand Y, Faigenboim-Doron A, Patil A, Hedvat I, Hovav R. Finemapping the branching habit trait in cultivated peanut by combining bulked segregant analysis and high-throughput sequencing. Front Plant Sci. 2017:8:467.

17. Dash S, Cannon EKS, Kalberer SR, Farmer AD, Cannon SB. PeanutBase and other bioinformatic resources for peanut (Chapter 8). In: Stalker HT, Wilson RF, editors. Peanuts genetics, processing, and utilization. AOCS press; 2016 p. 241-52.

18. Clevenger J, Chu Y, Scheffler B, Ozias-Akins P. A developmental transcriptome map for allotetraploid Arachis hypogaea. Front Plant Sci. 2016b;7.

19. Mizzotti C, Mendes MA, Caporali E, Schnittger A, Kater MM, Battaglia R, Colombo L. The MADS box genes SEEDSTICK and ARABIDOPSIS Bsister play a maternal role in fertilization and seed development. Plant J. 2012;70:409-20

20. Patil AS, Hedvat I, Levy Y, Galili S, Hovav R. Genotype-by-environment effects on the performance of recombinant inbred lines of Virginia-type peanut. Euphytica. 2018;214:83

21. Bradbury PJ, Zhang Z, Kroon DE, Casstevens TM, Ramdoss Y, Buckler ES. TASSEL: software for association mapping of complex traits in diverse samples. Bioinformatics. 2007;23:2633-5.

22. Bertioli DJ, Cannon SB, Froenicke L, Huang GD, Farmer AD, EKS C, et al. The genome sequences of Arachis duranensis and Arachis ipaensis, the diploid ancestors of cultivated peanut. Nat Genet. 2016;48:438-44.

23. Bonferroni CE. Teoria statistica delle classi e calcolo delle probabilita. Libreria Internazionale Seeber: Florence; 1936.
24. Van Ooijen JW, Voorrips RE. In: Kyazma BV, editor. JoinMap version 4.1 software for the calculation of genetic linkage maps. Wageningen: Plant research international; 2001.

25. Jansen J, De Jong AG, Van Ooijen JW. Constructing dense genetic linkage maps. Theor Appl Genet. 2001;102:1113-22.

26. Van Ooijen JW. In: Kyazma BV, editor. MapQTL 6 software for the mapping of quantitative trait loci in experimental populations. Wageningen: Plant research international; 2004.

27. Panse VG, Sukhatme PV. Statistical methods for agricultural workers. ICAR publication (2nd Ed.), New Delhi: 1985.
Ready to submit your research? Choose BMC and benefit from:

- fast, convenient online submission

- thorough peer review by experienced researchers in your field

- rapid publication on acceptance

- support for research data, including large and complex data types

- gold Open Access which fosters wider collaboration and increased citations

- maximum visibility for your research: over $100 \mathrm{M}$ website views per year

At $\mathrm{BMC}$, research is always in progress.

Learn more biomedcentral.com/submissions 